\title{
HIGH-RESOLUTION NIR IMAGING OF CIRCUMNUCLEAR REGIONS IN BARRED GALAXIES
}

\author{
D. PÉREZ-RAMÍREZ, J.H. KNAPEN \\ Department of Physical Sciences, University of Hertfordshire, \\ Hatfield, Herts AL10 9AB, UK \\ E-mail dperez,knapen@star.herts.ac.uk
}

We present preliminary results of our ongoing programme to obtain subarcsec resolution near-infrared (NIR) images and colour maps of the central regions of a sample of barred galaxies with circumnuclear starbursts, using the CFHT. In those cases where structure is seen in the broad band images, the NIR colour index maps confirm the location of dust lanes and regions of star formation. In those galaxies where the NIR broad band images appear featureless, the colour maps do show evidence for tightly wound spiral or ring structure, as hinted at in the optical.

\section{Introduction}

Bars or related structures are prevalent in galaxies. A galactic bar can be highly efficient in driving gaseous material into the central kpc of a galaxy (Shlosman et al. 1990; Athanassoula 1992; Phinney 1994).

Circum-nuclear rings delineated by star formation activity, molecular bars and inner stellar bars have all been observed, and appear to be associated with ILRs on scales of $1 \mathrm{kpc}$ (Knapen et al., 1995a). Several studies have outlined the relation between such regions and the structure of the host galaxies (Athanassoula 1992; Knapen et al. 1995a, 1995b; Knapen 1996). This relation has important implications for our understanding of the central activity, gas inflow processes and the galaxy structure.

\section{NIR Observations}

Our programme galaxies were observed with the MOntreal Near Infrared CAmera (MONICA; Nadeau et al. 1994) on the Canada France Hawaii Telescope (CFHT) between November 1995 and February 1996. 
The seeing in all cases was around 0.7 arcsec. The typical size of images is just over 1 arcmin.

We have obtained $J, H$ and $K$-band images, and have produced colour index maps by combining pairs of images. These colour images were created by dividing the two individual images, after removal of sky background. The colour index maps highlight changes in the stellar populations, as well as localized concentrations of dust.

\section{Results and discussion}

In general, the NIR colour index images show more structure than NIR broad band images. In most cases, we observe straight dust lanes coming into the circumnuclear region from the main bar, spiral shapes indicating miniature spirals arms accompanied by dust lanes, and "blue" sites of strong star formation close to the nucleus. In all cases, the NIR imaging reproduces the star formation structure as seen in the optical/ $\mathrm{H} \alpha$.

In a few other cases we observe that, whereas the broad-band NIR images are remarkably smooth and do not show the optical/ $\mathrm{H} \alpha$ structure in the circumnuclear region, the colour index maps do show such structure (e.g. NGC 4321), probably outlining spiral armlets accompanied by dust lanes.

In NGC 5248, the circumnuclear star formation activity shows up clearly in the broad-band NIR images, as well as in the colour index maps. It is clear that spiral structure, with star-forming arms accompanied by dust lanes, is present on all scales. We have recently obtained adaptive optics NIR images at a resolution of around 150 mas of this object which show that the spiral structure in fact continues to as close as 10 parsec from the nucleus.

These images will be combined with kinematic observations and dynamical modelling in order to understand the dynamics and evolution of barred galaxies.

\section{References}

Athanassoula, E. 1992. MNRAS 259, 345

Knapen, J.H. 1996, in : Barred galaxies and circumnuclear activity, Eds. A. Sandquist, P.O. Lindblad, Springer, 233

Knapen, J.H., Beckman, J.E., Shlosman, Peletier, Heller \& de Jong 1995a, ApJL 443, L73

Knapen, J.H., Beckman, J.E., Heller, Shlosman \& de Jong 1995b, ApJ 454, 623

Nadeau, D., Murphy, D.C., Doyon, R. \& Rowlands, N. 1994, PASP 106, 909

Phinney, E.S. 1994, in Mass-Transfer Induced Activity in Galaxies, ed. I. Shlosman, Cambridge: Cambridge Univ. Press, 1

Shlosman, I., Begelman, M.C. \& Frank, J. 1990, Nat. 345, 679 